\title{
Modeling Superconducting Transmission Line Bends and Their Impact on Nonlinear Effects
}

\author{
Jordi Mateu, Member, IEEE, Carlos Collado, Member, IEEE, and Juan M. O'Callaghan, Senior Member, IEEE
}

\begin{abstract}
This paper reports on a numerical technique to obtain the current distribution in the annular bent sections of planar layouts. This is used to obtain the linear and nonlinear circuit distributed parameters modeling a superconducting strip bend and its impact on intermodulation distortion. As an example, we analyze a superconductive open-loop resonator and assess the linear and nonlinear contribution of its bends in its overall linear and nonlinear performance. These simulations are very useful for optimizing the resonators of a filter in order to minimize its nonlinear distortion.
\end{abstract}

Index Terms-Circuit model, current distribution, nonlinear effects, superconductor, transmission line.

\section{INTRODUCTION}

$\mathbf{L}$ OW LOSSES of high temperature superconductive (HTS) thin films allow the fabrication of very compact and highperformance microwave filters [1], [2]. These planar devices often include narrow strip topologies with numerous multicoupled transmission lines [3] and bends. This usually leads to high current densities in the superconductor even at low input power [4], which not only affects the linear response of the filter, but may also give rise to undesirable nonlinear effects like intermodulation distortion (IMD) [5], [6]. An accurate modeling of these bends, particularly the current distribution in their cross section, is thus necessary to be able to model the linear and nonlinear response of superconducting devices, especially filters.

In straight transmission lines, there are well-known procedures to find the current distribution in the cross section of the line and calculate its inductance and resistance per unit length $(L, R)$ from it [7]-[9]. If the line is made from superconductor materials, this current distribution is known to change with the current through the line due to the properties of the superconductor (i.e., the current dependence of its penetration depth) [10]. This gives rise to a dependence of $L$ and $R$ on the current

\footnotetext{
Manuscript received September 14, 2006; revised January 11, 2007. This work was supported in part under the Fulbright Program and by the Spanish Government (CICYT) under Grant MAT-2005-05656-C03 and Grant TEC-2006-13248-C04-02/TCM and under the Ramón y Cajal Program through RyC-001125.

J. Mateu is with the Department of Signal Theory and Communications, Universitat Politècnica de Catalunya, Barcelona 08034, Spain, and also with the Centre Tecnològic de Telecomunicacions de Catalunya, Universitat Politècnica de Catalunya, 08860-Castelldefels, Barcelona, Spain (e-mail: jmateu@tsc.upc. edu).

C. Collado and J. M. O'Callaghan are with the Department of Signal Theory and Communications, Universitat Politècnica de Catalunya, Barcelona 08034, Spain (e-mail: collado@tsc.upc.edu; joano@tsc.upc.edu).

Color versions of one or more of the figures in this paper are available online at http://ieeexplore.ieee.org.

Digital Object Identifier 10.1109/TMTT.2007.895166
}

of the line, which provokes nonlinear effects. The calculation of the dependence of the current distribution on total current and its effects on $L$ and $R$ are well established for straight superconducting transmission lines [6], [10], but not for bent segments of lines.

The goal of this paper is to fill in this void, i.e., adapt the methods used in straight transmission lines to find the current distribution in a cross section of an annular bent transmission line, find its impact on $L$ and $R$, and in the case of superconducting lines, find how $L$ and $R$ depend on current and how this impacts IMD in a typical resonator that could be used in a superconducting filter.

In Section II, we describe the Weeks-Sheen method [7], [8] used to calculate $R$ and $L$ in normal and superconducting straight transmission lines and our extension for the linear modeling of annular bent sections. We will refer to the latter as the radial Weeks-Sheen method. Although this method is actually applied to annular bent sections, we will use the single term bend to refer to them throughout this paper. We also describe a cross check of this method where we analyze a copper microstrip bend and compare our results with those obtained with two alternative methods of the inductance per unit length in a normal conductor. Section III shows how to consider the nonlinear effects existing in superconducting materials using the distribution of the current density in a cross section of the bend obtained from the radial Weeks-Sheen method. Finally, in Section IV, we use this approach to predict the effects of bends in the linear and nonlinear response of a half-wave square-shaped open-loop resonator.

\section{WeEKS-SheEN Method For CuRVED TRANSMISSION LINES}

\section{A. Theoretical Background}

To evaluate the resistance and inductance per unit length of the strip, i.e., $R$ and $L$, one needs to know the volume current density distribution over the cross section of the line. This can be done using the Weeks et al. method [7], later modified by Sheen et al. [8] for superconductive transmission lines. This section shows the basics of the Weeks-Sheen method to illustrate how it is modified to be able to undertake the analysis of bent regions.

The cross section of the strip is meshed in smaller transmission lines resulting in a system of coupled transmission line equations. Fig. 1(a) shows a schematic diagram illustrating the meshing of a straight elemental segment of length $d z$. The meshing distribution is usually performed based on a priori intuition of the current distribution profile; i.e., choosing the smallest patches where the current distribution changes sharply 


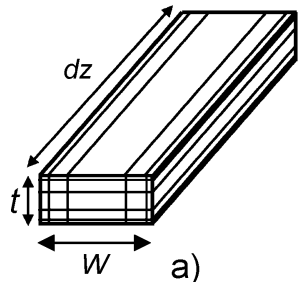

a)

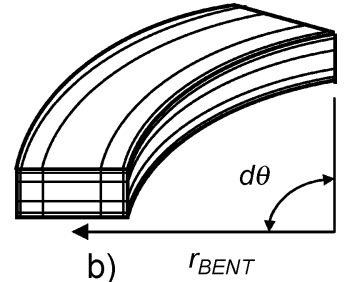

b) $\quad r_{B E N T}$

Fig. 1. Meshing of the cross section of the strip. (a) For a straight segment of the strip. (b) For a bent segment of the strip. $r_{\text {BENT }}$ defines the curvature of the bend, $w$ and $t$ define the width and thickness of the strip, respectively.

[9], this reduces the required number cells of the meshing and, therefore, reduces the computation time.

The resulting multicoupled transmission lines should satisfy the telegrapher's equation [7]

$$
\frac{d \bar{v}}{d z}=-\overline{\bar{Z}} \cdot \bar{i}
$$

where $\bar{v}$ is the vector containing the variation of the voltage of each patch relative to a reference patch-usually located in ground plane-as a function of the length of the segment $z$, and $\bar{i}$ is the vector containing the current in each line. $\bar{Z}$ is the matrix of self and mutual impedances per unit length between patches

$$
\overline{\bar{Z}}=\overline{\bar{R}}+j \omega \overline{\bar{L}}
$$

with $\overline{\bar{R}}$ and $\overline{\bar{L}}$ being resistances and inductances per unit length. The matrices may be calculated following the procedure discussed in detail by [8] (or [7] for normal conductors). Here, we just point out the expressions used to calculate the elements of $\overline{\bar{R}}$ and $\overline{\bar{L}}$. We write the elements in these matrices as $r_{m n}$ and $l_{m n}$, respectively, where $m$ and $n$ indicate the corresponding row and column. The resistive elements are given by

$$
r_{m n}=\operatorname{Re}\left(\frac{1}{\sigma_{0} S_{0}}+\delta_{m n} \frac{1}{\sigma_{m} S_{m}}\right)
$$

where $\delta_{m n}=1$ for $m=n$ and 0 for $m \neq n, S_{i}$ and $\sigma_{i}$ indicate the area and the complex conductivity of the patch $i$ th. The complex conductivity can be written as $\sigma=\sigma_{1}-j \sigma_{2}$ (where $\sigma_{2}=\left(\omega \mu_{0} \lambda_{L}^{2}\right)^{-1}, \lambda_{L}$ being the superconducting penetration depth). The calculation of $l_{m n}$ is somehow more complicated and can be split in a kinetic inductance $\left(l_{m n}^{k}\right.$, only existing in the superconducting case) and a partial inductance $\left(l_{m n}^{p}\right)$. The partial inductance includes the internal and external inductance corresponding, respectively, to the energy stored inside and outside of each conductor segment, due to the magnetic field, and can be obtained from [8, eqs. (11) and (12)]. The kinetic inductance can be obtained from the imaginary part of the superconducting impedance as [8]

$$
l_{m n}^{k}=\frac{1}{\omega} \operatorname{Im}\left(\frac{1}{\sigma_{0} S_{0}}+\delta_{m n} \frac{1}{\sigma_{m} S_{m}}\right) .
$$

As done in [8], we assume that the line voltages $\bar{v}$ are quasistatic, thus the voltages in the patches of the signal line are set to a constant value $v_{p}$ and 0 for patches of the ground plane. From a practical point-of-view, this implies that the term $d \bar{v} / d z$ takes a constant value for the patches in the signal strip and is 0 for

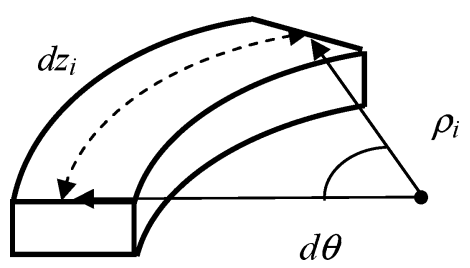

Fig. 2. Outline of one individual patch segment corresponding to a bent segment of the structure of Fig. 1(b).

the ground plane patches. Thus, (1) may be solved by inverting the impedance matrix

$$
\bar{i}=-\overline{\bar{Y}} \cdot \frac{d \bar{v}}{d z}
$$

with $\overline{\bar{Y}}$ being the admittance matrix $\left(\overline{\bar{Y}}=\overline{\bar{Z}}^{-1}\right)$. This gives us the current flowing through each line, which may be used to calculate the current density distribution $j$. By an algebraic addition of the elements from the admittance matrix corresponding to the signal line, that are in parallel, one obtains the admittance of the line and, thus, its inductance $(L)$ and resistance $(R)$ per unit of length [8].

\section{B. Radial Weeks-Sheen Method}

The purpose here is to modify the conventional Weeks-Sheen method to obtain the distributed parameters describing a bent elemental segment. Fig. 1(b) shows a schematic of a meshed bent elemental segment. Unlike the straight elemental segment of Fig. 1(a), in a bent region [see Fig. 1(b)], the elemental length $d z$ may be different for each line resulting from the meshing. We use, therefore, the angle $d \theta$ to define the best elemental sement of Fig. 1(b).

To analyze this structure, we first begin by considering a single segment of the meshed region. Fig. 2 outlines the $i$ th segment. The length of this segment is $d z_{i}$ and can be related with the angle defining the bent region $d \theta$ and the radius of the $i$ th patch segment $\rho_{i}$ as $d z_{i}=\rho_{i} \cdot d \theta$.

Considering the geometrical parameters defining each of the segments of a bend, (1) can be rewritten as

$$
\frac{d v_{i}}{d z_{i}}=-\sum_{j=1}^{N} Z_{i, j} \cdot i_{j}
$$

where $d v_{i}$ is the voltage drop in a segment of $d z_{i}$ length and $i_{j}$ is the current flowing through the $j$ th segment. The total number of segments $N$ is defined by the meshing. By considering $d z_{i}=$ $\rho_{i} \cdot d \theta,(6)$ can then be written as

$$
\frac{d v_{i}}{d \theta}=-\rho_{i} \sum_{j=1}^{N} Z_{i, j} \cdot i_{j}
$$

which in matrix form is

$$
\frac{d \bar{v}}{d \theta}=-\operatorname{diag}(\bar{\rho}) \cdot \overline{\bar{Z}} \cdot \bar{i}
$$

where the matrix $\operatorname{diag}(\bar{\rho})$ is diagonal and $\bar{\rho}$ is a vector containing the radius of each segment of the bend $\left(\bar{\rho}=\left[\rho_{1} \rho_{2} \cdots \rho_{N}\right]\right)$. Note that since the cross section of a straight elemental segment 
[see Fig. 1(a)] is equal to the cross section of a bent elemental segment [see Fig. 1(b)], the values of the matrix $\overline{\bar{Z}}$ should also be equal. Thus, we can define the equivalent matrix, which characterizes the cross section of the bent segment as

$$
\overline{\bar{Z}}_{\mathrm{BENT}}=\operatorname{diag}(\bar{\rho}) \cdot \overline{\bar{Z}} \text {. }
$$

The resulting equation of a multicoupled bent transmission lines is

$$
\frac{d \bar{v}}{d \theta}=-\overline{\bar{Z}}_{\mathrm{BENT}} \cdot \bar{i}
$$

By following the same procedure used to solve (1), we may obtain the current flowing through each patch of the bent segment from (10) as follows:

$$
\bar{i}=-\overline{\bar{Y}}_{\mathrm{BENT}} \cdot \frac{d \bar{v}}{d \theta}
$$

where $\overline{\bar{Y}}_{\mathrm{BENT}}$ is the equivalent admittance matrix of a bent region and the term $d \bar{v} / d \theta$ is constant for each segment belonging to the signal strip. From $\overline{\bar{Y}}_{\mathrm{BENT}}$, we obtain the inductance $\left(L_{\theta}\right)$ and resistance $\left(R_{\theta}\right)$ of the bent transmission line per unit angle. The inductance and resistance per unit length $L$ and $R$ can be straightforwardly obtained by dividing $L_{\theta}$ and $R_{\theta}$ by the radius defining the curvature of the bend $\left(r_{\mathrm{BENT}}\right) . r_{\mathrm{BENT}}$ is defined from the middle of the bend, thus its minimum value would be $0.5 \cdot w$.

\section{Cross-Check for a Normal Conductor Bend}

Here, we apply the radial Weeks-Sheen method for a normal conductor to be able to compare the results with existing techniques. This can be done by considering a real conductivity [7], which allows us to neglect (4) in the impedance matrix calculation. The microstrip bend has a cross section with $0.5 \mathrm{~mm}$ of width and $0.43 \mathrm{~mm}$ of substrate thickness.

Two different approaches, contained in a commercial software package [12], have been used to obtain the inductance per unit length of a copper bend as a function of its radius. The first approach consists of a microstrip circuit model for curved bends based on perturbation techniques [13]. Its results are shown in the squares in Fig. 3. In this case, we see how the effects of the bend start for $r_{\mathrm{BENT}} / w \approx 4$, smoothly reducing the distributed inductance as the radius decreases. The second approach performs electromagnetic simulation of the planar structure by using techniques based on the method of moments [12]. The results are shown in triangles in Fig. 3. Finally, the dashed line in Fig. 3 shows the results using the method we propose. The latter two methods show very good agreement for the whole range of $r_{\mathrm{BENT}} / w$ and predict a weak dependence of $L$ on $r_{\mathrm{BENT}}$ for values of $r_{\mathrm{BENT}} / w>2$ and a sharp decrease for smaller values of $r_{\mathrm{BENT}} / w$.

\section{NONLINEAR SUPERCONDUCTING BENDS}

\section{A. Calculation of the Nonlinear Parameters}

In a superconducting case, the nonlinear dependence of the superfluid density on the current density $j_{s}$ gives rise to a nonlinear complex conductivity [6], $\sigma\left(T, j_{s}\right)=$

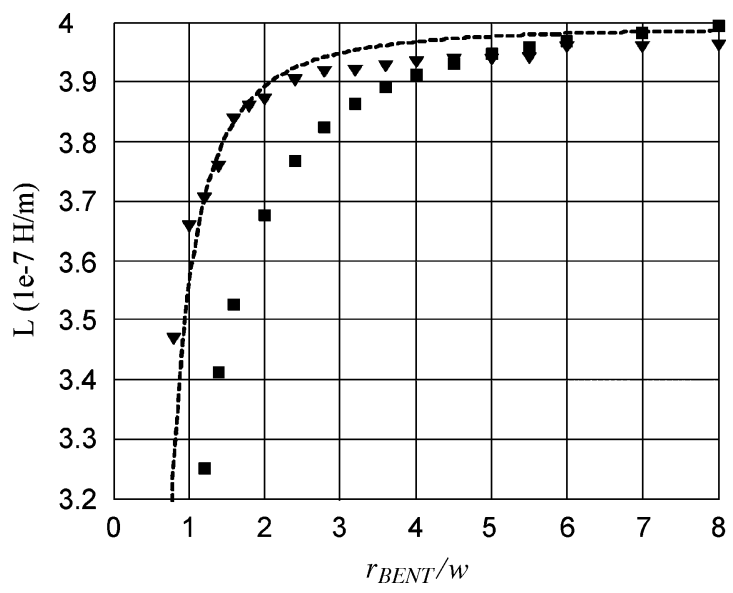

Fig. 3. Variation of the distributed inductance of a cooper microstrip bend $(L)$ as a function of $r_{\text {BENT. }}$. Squares represent the results obtained using the circuit model based on [13], triangles correspond to the full-wave simulation results and the dashed line corresponds to the simulation with our technique.

$\sigma_{1}\left(T, j_{s}\right)-j / \omega \mu_{0} \lambda_{L}^{2}\left(T, j_{s}\right)$. In this equation, the conductivity of normal fluid $\sigma_{1}(T, j)$ and the penetration depth of the supercondutor $\lambda_{L}(T, j)$ depend on temperature and current density as

$$
\begin{aligned}
\sigma_{1}\left(T, j_{s}\right) & =\sigma_{1}(T, 0)\left[1+a(T) f\left(T, j_{s}\right)\right] \\
\lambda_{L}^{2}\left(T, j_{s}\right) & =\lambda_{L}^{2}(T, 0)\left[1-f\left(T, j_{s}\right)\right]^{-1}
\end{aligned}
$$

where the function $f(T, j s)$ describes the form of the nonlinearity and $a(T)$ relates the relative magnitudes of the real and imaginary components of the nonlinear conductivity [6].

To evaluate these deviations, we use an iterative procedure [10], which, from the current distribution of the current iteration updates $\lambda_{L}\left(j_{s}\right)$ and $\sigma_{1}\left(j_{s}\right)$ of the next iteration using (12). From these new values, we recalculate the current distribution and repeat this procedure until convergence is achieved. By running this procedure for several values of voltage in the signal strip $v_{p}$, we determine the nonlinear current dependence of the inductance $L(T, i)$ and resistance $R(T, i)$ per unit of length. Note that the nonlinear current dependence of the distributed inductance is only due to the variation of the kinetic part of the inductance.

We assume a quadratic nonlinear dependence of the superfluid density on the current density, i.e., $f(T, j)=\left(j / j_{2}(T)\right)^{2}$ $\left(j_{2}(T)\right.$ being a characteristic current density that sets the strength of nonlinearities), which is a very good approximation for weak nonlinear effects $j_{s} \ll j_{2}$ [10]. In this case, the resulting distributed parameters $[L(T, i)$ and $R(T, i)]$ can be obtained from closed-form equations and also follow a quadratic dependence on the current flowing through the line

$$
\begin{aligned}
& L(T, i)=L_{0}(T)+\Delta L(T) \cdot i^{2} \\
& R(T, i)=R_{0}(T)+\Delta R(T) \cdot i^{2}
\end{aligned}
$$

where the nonlinear terms $\Delta L(T)$ and $\Delta R(T)$ can be found from the following expressions [14]:

$$
\begin{aligned}
\Delta L(T) & =\frac{\mu_{0} \lambda_{L}^{2}(T, 0)}{j_{2}^{2}(T)} \Gamma^{\prime}(T) \\
\Delta R(T) & =\sigma_{1}(T, 0) \omega^{2} \mu_{0}^{2} \lambda_{L}^{4}(T, 0) \frac{2+a(T)}{j_{2}^{2}(T)} \Gamma^{\prime}(T)
\end{aligned}
$$


where $\Gamma^{\prime}$ is a geometrical factor, which depends on the current density distribution over the cross section

$$
\Gamma^{\prime}(T)=\frac{\int j_{s}^{4} d S}{\left(\int j_{s} d S\right)^{4}}
$$

The nonlinear dependence of the distributed resistance and inductance using (13)-(15) has been verified using the above outlined iterative procedure [10]. This procedure may show divergence for high current densities or strong nonlinear effects. Although the range of validity may be improved using a more robust iterative procedure, we estimate the validity of this method for $j_{s}<0.1 \cdot j_{2}$.

In Section III, we will evaluate these quantities for a straight and bent segment of a strip, such as the ones shown in Fig. 1.

\section{B. Modeling of a Microstrip Bend}

Here, we use the above-described procedure to obtain the linear and nonlinear distributed parameters $(L(i)$ and $R(i))$ in a microstrip superconducting bent transmission line as a function of its radius.

The cross section used for this example is a microstrip structure where the width $(w)$ of the signal line is $0.5 \mathrm{~mm}$, the thickness $(t)$ of the superconducting strip and ground plane is $270 \mathrm{~nm}$, and the thickness of the dielectric substrate is $0.43 \mathrm{~mm}$. The material is YBCO on $\mathrm{MgO}$. The surface resistance of the material at $77 \mathrm{~K}$ and $10 \mathrm{GHz}$ is $0.7 \mathrm{~m} \Omega$ and the penetration depth at $77 \mathrm{~K}$ is $230 \mathrm{~nm}$. The simulations are performed at $2 \mathrm{GHz}$ since it is a frequency of interest in wireless communication applications. Note that the topology of the structure and properties of the material considered for this simulation are commonly used in superconducting filter designs [15].

Fig. 4 depicts the current density distribution in the cross section for a straight transmission line [see Fig. 4(a)] and for a bent transmission line with $r_{\mathrm{BENT}} / w=0.8$ [see Fig. 4(b)]. The current density distribution in a straight line segment has a symmetric profile, whereas in the bent segment, as we expect, the current density distribution is higher at the inner part. As we will show below, this has consequences on both the linear and nonlinear parameters defining the circuit model of the line.

To evaluate the effects of the bend in the linear parameters defining the bent transmission line, Fig. 5 shows the linear inductance $\left(L_{b}\right)$ and resistance $\left(R_{b}\right)$ per unit length as a function of the ratio between the radius of the bend and the width of the line $\left(r_{\mathrm{BENT}} / w\right)$. Note also that $L_{b}$ and $R_{b}$ in Fig. 5 are normalized by the inductance $\left(L_{s}\right)$ and resistance $\left(R_{s}\right)$ of a straight segment. These results show a reduction of the inductance and an increment of the resistance when the radius decreases. Note that, to guarantee a less than $10 \%$ deviation with respect to the straight-line values of $L_{s}, r_{\mathrm{BENT}} / w$ should be kept above 1 . This condition is slightly more stringent for $R_{s}\left(r_{\mathrm{BENT}} / w>1.5\right)$.

We have also assessed the impact of the bends in the nonlinear performance of a superconducting transmission line. To do this, we assume the quadratic nonlinear behavior of Section II-A and determine how the geometric factor $\Gamma^{\prime}$ changes with $r_{\mathrm{BENT}} / w$.

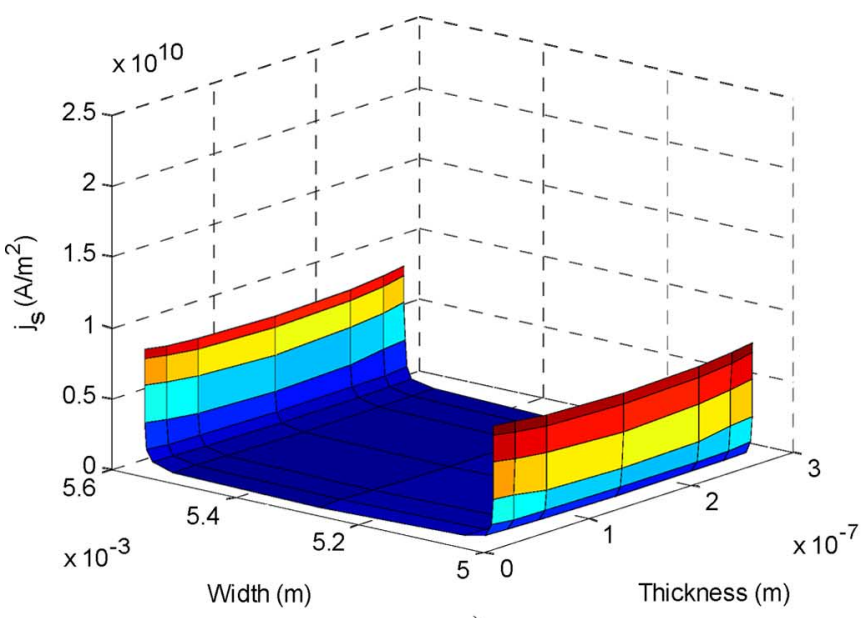

a)

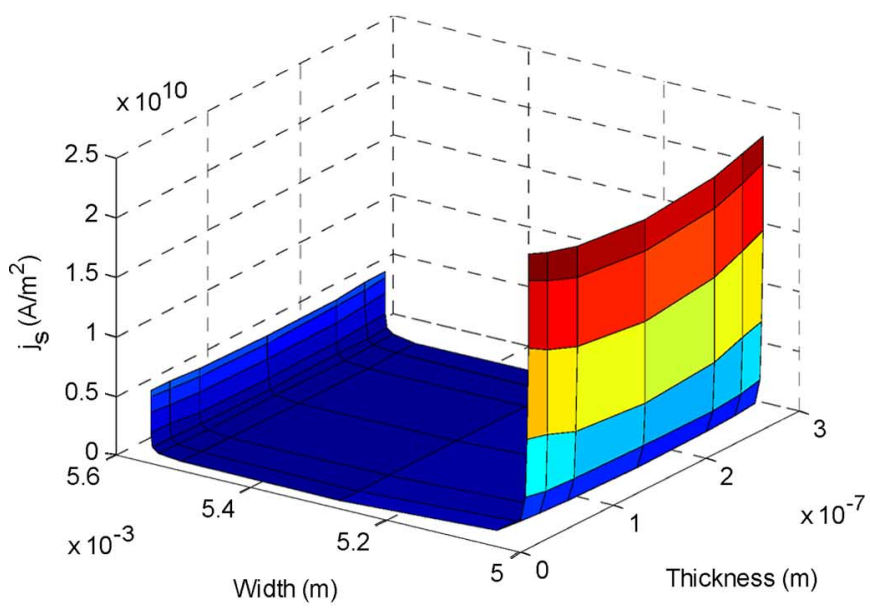

b)

Fig. 4. Volume current density distribution over the signal strip of microstrip topology. (a) For a straight elemental segment. (b) For a bent elemental segment with $r_{\mathrm{BENT}} / w=0.8$.

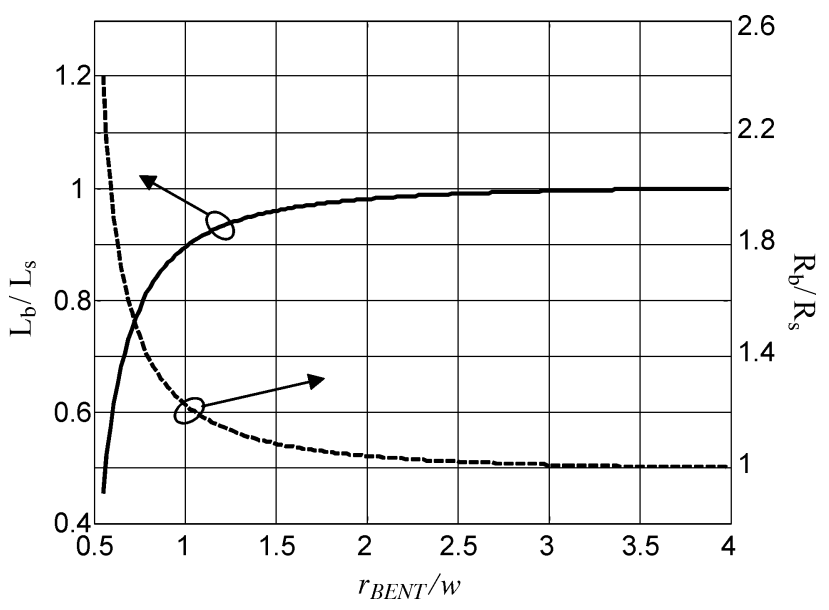

Fig. 5. (left) Variation of the distributed inductance of a bend $\left(L_{b}\right)$ as a function of $r_{\mathrm{BENT}}$. (right) Variation of the distributed resistance of a bend $\left(R_{b}\right)$ as a function of $r_{\mathrm{BENT}}$. Both are normalized by the distributed parameters in a straight segment, $L_{s}$ and $R_{s}$, respectively.

Fig. 6 depicts the dependence of $\Gamma^{\prime}$ on the radius of the bent segment. We see that the nonlinearities may increase by a factor of 20 when the radius gets close to $0.5 \cdot w$, which is likely to affect 


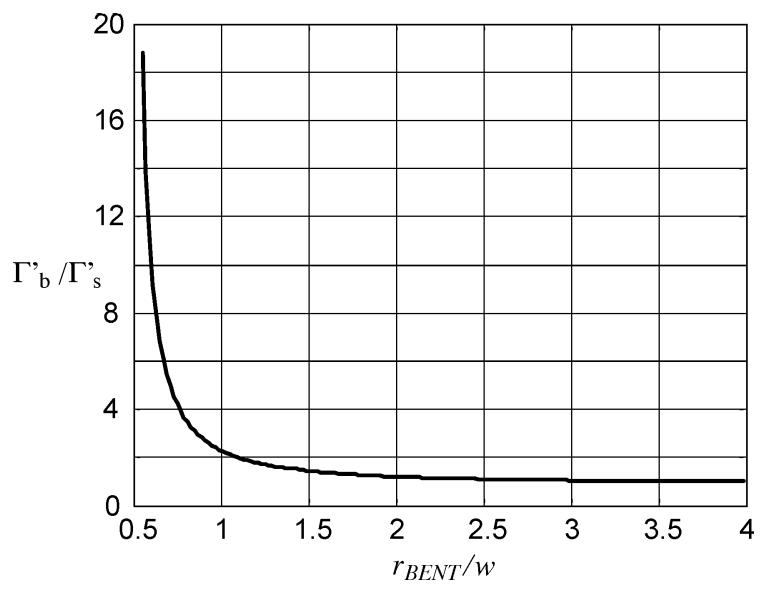

Fig. 6. Nonlinear geometrical factor $\Gamma^{\prime}(9)$ as a function of the radius of the bent segment normalized by $\Gamma^{\prime}$ in a straight segment.

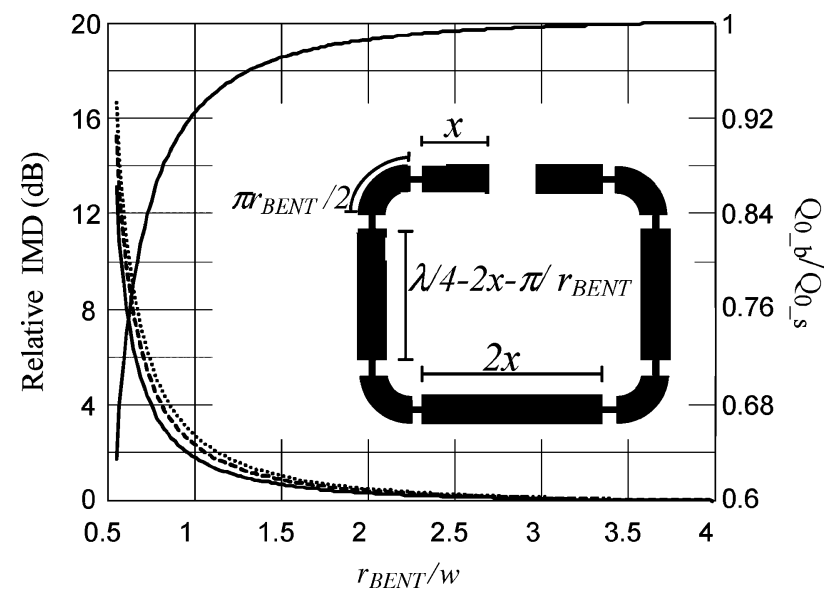

Fig. 7. Open-loop resonator IMD products (in decibels) as a function of the $r_{\mathrm{BENT}} / w$ normalized by the IMD in a straight resonator. Solid, dashed, and dotted lines correspond to $x=\lambda / 40, \lambda / 20$, and $\lambda / 10$, respectively. Variation of the normalized quality factor $\left(Q_{0_{-} b} / Q_{0_{-} s}\right)$ as a function of $r_{\mathrm{BENT}} / w$. Note that, in this layout, the effect of the gap length is neglected.

the overall nonlinear performance of the device containing the bend.

The effects shown above are very important from an engineering point-of-view since it is necessary to predict them for a proper design of resonator and filter topologies. Note also that this is relevant for materials characterization since many planar devices used to obtain linear and nonlinear parameters to characterize the superconducting materials use planar patterns containing bends [16], [17].

Here, it has been shown how the distributed parameters in a bent segment deviate from the ones expected in a straight segment. Section IV goes one step further, showing the application of these results to evaluate the effects of bent regions in practical microwave devices.

\section{OPEN-LOOP RESONATORS WITH BENDS}

Here, we analyze how the linear and nonlinear performance of an open-loop resonator is affected by the radius of the bend $\left(r_{\mathrm{BENT}}\right)$ and by the position where the bends are located. The inset of Fig. 7 outlines the topology of the open-loop under study. It contains four bends, two of them are placed at a dis- tance $x$ from the open ends of the resonator and the other two at a distance $x$ from the center. The cross section and parameters of the resonator are the ones used in Section IV-A. The length of the resonator has been adjusted to operate on its first resonant mode (i.e., half-wave resonator) at $2 \mathrm{GHz}$. That is the current distribution, which follows a sinusoidal distribution along the resonator.

To analyze this structure, we have split the resonator in straight regions and bent regions (see the inset of Fig. 7). The equivalent-circuit model of the whole resonator consists of concatenating many elemental $R L C G$ cells, corresponding to a straight or a bent region.

The equivalent circuit can now be solved either using a circuit analysis tool (note that it should be able to apply nonlinear analysis, such as harmonic-balance techniques [18]) or by developing the closed-form expression, which gives the IMD products generated along the resonator of length $l$. We have obtained this expression by following the procedure detailed in [6]. Unlike [6], in this case, we should consider the dependence of the circuit parameters $(L(i)$ and $R(i))$ on their location along the resonator.

To do that, we assume a spatial sinusoidal distribution of the fundamental and IMD frequencies. For quadratic nonlinearities [see (13)], the nonlinear voltage at IMD frequency in an elemental segment of the resonator is

$$
\frac{d V_{\mathrm{IMD}}(z)}{d z}=\frac{3}{4} I_{1}^{2} I_{2}^{*}(\Delta R(z)+j \omega \Delta L(z)) \sin ^{3}\left(\frac{\pi z}{l}\right)
$$

where $I_{1}$ and $I_{2}$ are the current of the fundamentals. Now the power generated at IMD frequency $0.5 \int_{0}^{l} I_{\mathrm{IMD}}^{*}(z)\left(d V_{\mathrm{IMD}}(z) / d z\right) d z$ will be dissipated in the resonator (dielectric losses are assumed negligible [9]) and coupling loads $0.5(1+2 \beta) \int_{0}^{l} R(z)\left|I_{\mathrm{IMD}}(z)\right|^{2} d z$, where $\beta$ is the coupling coefficient [19] and, thus, the term $2 \beta$ accounts for the dissipation on the input and output, assuming equal coupling. Note that these integrals should consider the value of the linear and nonlinear distributed parameter at each position of the resonator. Once we know $I_{\mathrm{IMD}}$, the power at the IMD frequency coupled to the load is

$$
P_{\mathrm{IMD}}=\frac{1}{4} \beta \int_{0}^{l} R(z)\left|I_{\mathrm{IMD}}(z)\right|^{2} d z .
$$

This expression has been verified by simulating the equivalent circuit of the whole resonator of the inset of Fig. 7, which consists of cascading many $R L C G$ elemental cells, using a circuit analysis simulator [12].

The results of this analysis are shown in Fig. 7. The right-hand axis indicates the quality factor of the half-wave open-loop resonator normalized by the quality factor in a half-wave straight resonator. The quality factor decreases when $r_{\mathrm{BENT}}$ decreases. We see that, for $r_{\mathrm{BENT}} / w=0.8$, the quality factor drops more than $10 \%$, and for $r_{\mathrm{BENT}} / w=0.6$, it degrades more than $30 \%$. The quality factor is barely affected by the position of the bents. The left-hand axis in Fig. 7 indicates the IMD of the open-loop resonator normalized by the IMD that occurs in a straight resonator. These results show how the nonlinearities rapidly increase when $r_{\mathrm{BENT}}$ decreases. When $r_{\mathrm{BENT}} / w=0.8$, the IMD 
increases more than $4 \mathrm{~dB}$, and for $r_{\mathrm{BENT}} / w=0.6$, it increases more than $10 \mathrm{~dB}$. As occurs with the quality factor, the IMD is not strongly affected by the position of the bends. This may be explained by assuming a sinusoidal distribution along the strip. When $x$ increases (or decreases), the two bends closer to the ends have a stronger (or weaker) contribution, whereas the other two bends have a weaker (or stronger) contribution. Note that these effects depend on the resonator topology.

The resonant frequency of the resonator would also be affected by the bent segments contained in the structure. This can be concluded from the deviation of the distributed inductance as a function of the radius in Fig. 3. However, the bends would also introduce an additional distributed capacitance [19], which will also affect the resonant frequency of the structures, thus we cannot obtain the frequency shift in the resonator only from the deviation of the inductance due to the bent segments. Note that this would not occur for the quality factor since the losses coming from the dielectric (which are also affected by the bent section) are negligible [6]. Although the frequency shift is a very important designing parameter, in practice, this can usually be tuned by making the resonator slightly longer or shorter, whereas the quality factor and IMD are parameters that strongly depend on the shape of the resonator (and material properties) and cannot be tuned for a given geometry.

\section{CONCLUSION}

The radial Weeks-Sheen method proposed in this paper has been shown to be consistent with other methods of analyzing normal conducting bends of planar microwave circuits. Unlike the methods used in the comparison, the radial Weeks-Sheen method is also applicable to superconductors and can be used to predict the linear and nonlinear effects of a bend. We have analyzed a typical microstrip geometry and we found that, to keep the inductance per unit length in the bend within $10 \%$ of its value in a straight line, $r_{\mathrm{BENT}} / w$ should be kept higher than 1 in both a superconducting and a normal-metal strip (Figs. 3 and $5)$. This condition is slightly more stringent for the resistance per unit length of a superconducting strip $\left(r_{\mathrm{BENT}} / w>1.5\right)$.

When analyzing the nonlinear effects of bends in an open-loop resonator at $2 \mathrm{GHz}$ (Fig. 7), we found that when $r_{\mathrm{BENT}} / w=1, Q$ decreases approximately $10 \%$ with respect to that of a straight-line resonator, and IMD increases by $2-3 \mathrm{~dB}$ depending on the position of the bends. In any case, both IMD and $Q$ degrade significantly for lower values of $r_{\mathrm{BENT}} / w$, which would make them inadequate for high-performance superconducting filters.

While this paper and its conclusions have an obvious relevance for microwave engineering purposes, they may also be of interest for testing superconductors since many test devices consist of planar circuits containing strip bends.

\section{ACKNOWLEDGMENT}

The authors would like to thank Dr. R. Taylor and R. Clarke, both with Microwave and Materials Designs Pty. Ltd., Brisbane, Australia, for fruitful discussions and comments.

\section{REFERENCES}

[1] J.-S. Hong and M. J. Lancaster, "Compact microwave elliptic function filter using novel microstrip meander open-loop resonators," Electron. Lett., vol. 32, pp. 563-564, 1996.

[2] H. Su and M. J. Lancaster, "Highly miniature HTS microwave filters," IEEE Trans. Appl. Supercond., vol. 11, no. 1, pp. 349-352, Mar. 2001.

[3] J. Mateu, C. Collado, and J. M. O'Callaghan, "Nonlinear model of coupled superconducting lines," IEEE Trans. Appl. Supercond., vol. 15, no. 2, pp. 976-979, Jun. 2005.

[4] D. E. Oates, S.-H. Park, D. Agassi, G. Koren, and K. Irgmaier, "Temperature dependence of intermodulation distortion in YBCO: Understanding nonlinearity," IEEE Trans. Appl. Supercond., vol. 15, no. 2, pp. 3589-3595, Jun. 2005.

[5] M. I. Salkola, "Nonlinear characteristics of a superconducting receiver," Appl. Phys. Lett., vol. 88, pp. 012501/1-012501/3, 2006.

[6] C. Collado, J. Mateu, and J. M. O'Callaghan, "Analysis and simulation of the effects of distributed nonlinearities in microwave superconducting devices," IEEE Trans. Appl. Supercond., vol. 15, no. 1, pp. 26-39, Mar. 2005.

[7] W. T. Weeks, L. L. Wu, M. F. McAllister, and A. Singh, "Resistive and inductive skin effect in rectangular conductors," IBM J. Res. Dev., vol. 23, pp. 652-660, 1979.

[8] D. M. Sheen, S. M. Ali, D. E. Oates, R. S. Whiters, and J. A. Kong, "Current distribution, resistance, and inductance for superconducting strip transmission lines," IEEE Trans. Appl. Supercond., vol. 1, no. 2, pp. 108-115, Jun. 1991.

[9] A. Porch, M. J. Lancaster, and R. G. Humphreys, "The coplanar resonator technique for determining the surface impedance of $\mathrm{YBaCO}$ thin film," IEEE Trans. Microw. Theory Tech., vol. 43, no. 2, pp. 306-314, Feb. 1995.

[10] T. Dahm and D. Scalapino, "Theory of intermodulation in superconducting microstrip resonator," J. Appl. Phys., vol. 81, no. 4, pp. 2002-2006, 1997.

[11] J. C. Booth, J. Bell, D. Rudman, L. Valle, and R. Ono, "Geometry dependence of nonlinear at microwave frequencies," J. Appl. Phys., vol. 86, no. 2, pp. 1020-1025, 1999.

[12] Advanced Design System. Agilent Technol., Palo Alto, CA, 2005.

[13] A. Weisshaar and V. K. Tripathi, "Perturbation analysis and modeling of curved microstrip bends," IEEE Trans. Microw. Theory Tech., vol. 38 , no. 10 , pp. $1449-1454$, Oct. 1990.

[14] J. C. Booth, K. Leong, S. A. Schima, C. Collado, J. Mateu, and J. M. O'Callaghan, "Unified description of nonlinear effects in high temperature superconductors," J. Supercond. 2006.

[15] J.-S. Hong, M. J. Lancaster, D. Jedamzik, and R. B. Greed, "On the development of superconducting microstrip filter for mobile communication applications," IEEE Trans. Microw. Theory Tech., vol. 47, no. 9, pp. 1656-1663, Sep. 1999.

[16] Y. Wang, H. T. Su, F. Huang, and M. J. Lancaster, "Wide-band superconducting coplanar delay line," IEEE Trans. Microw. Theory Tech., vol. 53, no. 7, pp. 2348-2354, Jul. 2005.

[17] Y. Wang, H. Su, F. Huang, and M. J. Lancaster, "Measurements of YBCO surface resistance using coplanar line resonator techniques from $20 \mathrm{MHz}$ to $20 \mathrm{GHz}$," IEEE Trans. Appl. Supercond., submitted for publication.

[18] S. A. Maas, Nonlinear Microwave Circuits. Boston, MA: Artech House, 1998.

[19] J.-S. Hong and M. J. Lancaster, Microstrip Filters for RF/Microwave Applications, ser. Microw. Opt. Eng. New York: Wiley, 2001.

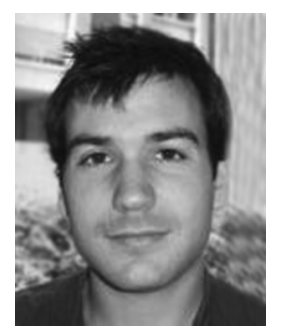

Jordi Mateu (M'03) was born in Llardecans, Spain, in 1975. He received the Telecommunication Engineering and $\mathrm{Ph} . \mathrm{D}$. degrees from the Universitat Politècnica de Catalunya (UPC), Barcelona, Spain, in 1999 and 2003, respectively.

Since October 2006, he has been Research Fellow with the Department of Signal Theory and Communications, UPC. From May to August 2001, he was Visiting Researcher with Superconductor Technologies Inc., Santa Barbara, CA. From October 2002 to August 2005, he was Research Associate with the Telecommunication Technological Center of Catalonia, Catalonia, Spain. Since September 2004, he has held several Guest Researcher appointments with the National Institute of Standards an Technology (NIST), Boulder, CO, where he was a Fulbright Research Fellow from September 2005 to October 2006 In July 2006, he was a Visiting Researcher with the Massachusetts Institute of Technology (MIT) Lincoln Laboratory. From September 2003 to August 
2005, he was a Part-Time Assistant Professor with the Universitat Autònoma de Barcelona. His primary research interests include microwave devices and system and characterization and modeling of new electronic materials including ferroelectrics, magnetoelectric, and superconductors.

Dr. Mateu was the recipient of the 2004 Prize for the best doctoral thesis in fundamental and basic technologies for information and communications presented by the Colegio Oficial Ingenieros de Telecomunicación (COIT) and the Asociación Española de Ingenieros de Telecomunicación (AEIT). He was also the recipient of a Fulbright Research Fellowship, an Occasional Lecturer Award for visiting MIT, and a Ramón y Cajal Contract.

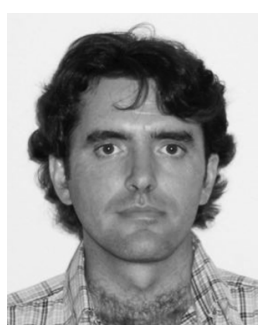

Carlos Collado (M'05) was born in Barcelona, Spain, in 1969. He received the Telecommunication Engineering sand Ph.D. degrees from the Technical University of Catalonia (UPC), Barcelona, Spain, in 1995 and 2001, respectively.

In 1998, he joined the faculty of UPC, where he has been teaching courses on the theory of electromagnetism, microwave laboratory, and high-frequency devices and systems. In 2004, he was a Visiting Researcher with the University of California at Irvine. Since April 2005, he has been an Associate Professor with UPC. His primary research interests include microwave devices and systems, electrooptics applications, and superconducting devices.

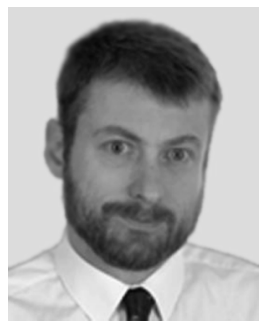

Juan M. O'Callaghan (SM'01) received the Telecommunication Engineering degree from the Universitat Politècnica de Catalunya (UPC), Barcelona, Spain, in 1987, and the M.S. and Ph.D. degrees from the University of Wisconsin-Madison, in 1989 and 1992, respectively.

He is currently a Full Professor with UPC. He was an intern with the Systems Research Center, Honeywell, Bloomington, $\mathrm{MN}$, where he was involved with noise measurement methods for field-effect transistors (FETs) at $K a$-band. From 2003 to 2006 he was Manager for MERIT, a consortium of European universities delivering a joint master's program in information technologies within the Erasmus Mundus Program. He is currently Vice-Dean of Academic Affaires with Telecom BCN, the telecommunication engineering school of UPC. He has authored or coauthored over 40 papers in peer-reviewed international magazines. He holds three patents. His research interests include microwave devices and materials and microwave photonics. He has been involved with noise characterization, large-signal properties of GaAs FETs, and advanced microwave materials such as superconductors and ferroelectrics. 